\title{
VASCULITIS INDUCED BY BIOLOGICAL AGENTS USED IN RHEUMATOLOGY: A SYSTEMATIC REVIEW
}

\author{
Camila da Silva Cendon Duran", , Adriane Souza da Paz², Mittermayer Barreto Santiago ${ }^{2}$ \\ 1. Universidade de São Paulo, São Paulo (SP), Brazil. 2. Universidade Federal da Bahia, Salvador (BA), Brazil. \\ *Corresponding author: milla_duran@hotmail.com
}

\section{BACKGROUND}

Biological medications have been used with increasing frequency to treat rheumatological diseases. These medications promise greater safety since they have well-defined biological targets. However, many induced autoimmune events have been described, such as vasculitis, that most often affects the skin (small-caliber vessels) and other organs. If diagnosed early, it can improve the prognosis, since drug withdrawal in most cases is sufficient to control the condition. This review aims to analyze the clinical profile of patients with vasculitis induced by biological agents.

\section{MATERIALS AND METHODS}

We performed a systematic review following the PRISMA recommendations. PubMed was used to search for eligible articles. We included case reports and case series of patients using anti-TNF therapy, tocilizumab, ustekinumab, secukinumab, rituximab, and abatacept diagnosed with drug-induced vasculitis. The following descriptors were used: "vasculitis" combined with "biological therapy”, "etanercept”, "infliximab”, "adalimumab”, "certolizumab”, "golimumab”, "rituximab”, "abatacept”, "tocilizumab”, "ustekinumab”, "secukinumab". Studies were selected based on titles and abstracts. Those that met the eligibility criteria were separated for a full review, and those which did not meet the eligibility criteria were excluded. Data extraction was performed using a standard form.

\section{RESULTS}

The initial database search identified 5361 articles, with 76 included for final analysis ( 80 patients), of whom 52 were women, median 47.5 years (range 15-82). Twenty-two patients were using adalimumab, 24 infliximab, 16 etanercept, 5 secukinumab, 4 certolizumab, 4 rituximab, 2 golimumab, 1 abatacept, 1 tocilizumab and 1 ustekinumab. Unspecific leukocytoclastic vasculitis (LCV) was the most common type of vasculitis (35) followed by ANCA-associated vasculitis (14), Henoch-Schonlein purpura (12), Takayasu arteritis (4), aortitis (2), giant cell arteritis (2), Behçet's disease (2), cutaneous medium-sized vessel vasculitis (2), hypocomplementemic urticarial vasculitis (1), lymphocytic vasculitis (1), retinal vasculitis (1), erythema elevatum diutinum (1), polyarteritis nodosa (1), pulmonary vasculitis (1) and peripheral neuropathy secondary to necrotizing vasculitis (1). The time frame from the start of therapy and the onset of vasculitis ranged from hours to 10 years. In 13 cases, the biological therapy was maintained, but 8 cases recurred, and it was necessary to remove the drug. The medication was changed in 16 cases, with only three relapses.

\section{CONCLUSION}

Infections, infusion reaction, cancer, and autoimmune events are well-known side effects of biological therapy. This review demonstrated that vasculitis is another adverse effect of this kind of therapy, particularly the anti-TNF-alpha molecules, and LCV the most reported type of vasculitis. 